\title{
Modelo de rendimiento de microtuneladoras (MTBM)
}

\section{Performance model for Micro Tunnelling Boring Machines (MTBM)}

\author{
J. Gallo $^{(*)}, \underline{\text { H. Pérez-Acebo }}{ }^{(* *)}$
}

\section{RESUMEN}

Desde las últimas décadas del siglo XX se han propuesto diversas fórmulas para estimar el rendimiento de la excavación de túneles mediante el uso de discos cortadores, empleados principalmente en tuneladoras, Tunnelling Boring Machines (TBM). Sin embargo, no se ha verificado su validez en microtuneladoras, Micro Tunnelling Boring Machines (MTBM), de menor diámetro de excavación, entre 1.000 y $2.500 \mathrm{~mm}$ y de útiles de corte más pequeños, donde parámetros como el espaciamiento entre juntas pueden tener una influencia distinta. Este artículo analiza esos modelos propuestos para TBM y, tras observar su escasa correlación con los datos obtenidos en 15 tramos de microtúneles, desarrolla un modelo ajustado a los parámetros geomecánicos disponibles en este tipo de obras. Además, se presenta un método para el cálculo del número de horas totales necesarias para la realización de microtúneles, incluyendo todas las tareas del ciclo de excavación, la instalación y la retirada.

Palabras clave: microtuneladora; modelo de rendimiento; disco cortador; field penetration index (FPI); excavación de túneles.

\section{ABSTRACT}

From the last decades of the XX century, various formulae have been proposed to estimate the performance in tunnelling of disc cutters, mainly employed in Tunnelling Boring Machines (TBM). Nevertheless, their suitability has not been verified in Micro Tunnelling Boring Machines (MTBM), with smaller diameter of excavation, between 1,00O and 2,500 mm and smaller cutter tools, where parameters like joint spacing may have a different influence. This paper analyzes those models proposed for TBM. After having observed very low correlation with data obtained in 15 microtunnels, a new performance model is developed, adapted to the geomechanical data available in this type of works. Moreover, a method to calculate the total amount of hours that are necessary to carry out microtunnels, including all the tasks of the excavation cycle and installation and uninstallation.

Keywords: Micro Tunnelling Boring Machine; performance model; disc cutter; field penetration index (FPI); tunnelling.

(*) Departamento de Ingeniería Minera, Metalurgia y Ciencia de los Materiales, Universidad del País Vasco UPV/EHU, Bilbao (España).

(**) Departamento de Ingeniería Mecánica, Universidad del País Vasco UPV/EHU, Bilbao (España).

Persona de contacto/Corresponding author: heriberto.perez@ehu.eus (H. Pérez-Acebo)

ORCID: http://orcid.org/oooo-0oo1-9890-0610 (J. Gallo); http://orcid.org/oooo-0003-0577-9597 (H. Pérez-Acebo)

Cómo citar este artículo/Citation: Gallo, J., Pérez-Acebo, H. (2017). Modelo de rendimiento de microtuneladoras (MTBM). Informes de la Construcción, 69(546): e203, doi: http://dx.doi.org/10.3989/id55211.

Copyright: (c) 2017 CSIC. Licencia / License: Salvo indicación contraria, todos los contenidos de la edición electrónica de Informes de la Construcción se distribuyen bajo una licencia de uso y distribución Creative Commons Attribution License (CC BY) Spain 3.o. 


\section{INTRODUCCIÓN}

El proceso de excavación en roca mediante el uso de discos cortadores se ha analizado desde los años 70 del siglo XX y se han desarrollado distintos modelos a partir de estudios teóricos o empíricos para estimar su funcionamiento (1) (2). Los modelos teóricos se desarrollan a partir de las fuerzas que actúan sobre los discos cortadores, mientras que los empíricos se basan principalmente en las observaciones de los rendimientos obtenidos de las máquinas en la realidad. De esta manera los parámetros empleados en estos modelos se pueden clasificar en dos grandes grupos:

a) Los modelos empíricos se basan principalmente en las observaciones de los rendimientos obtenidos por las máquinas in situ y precisan únicamente parámetros geomecánicos de la roca intacta; como la resistencia a compresión, $\sigma_{C}$, resistencia a tracción mediante el ensayo brasileño, $\sigma_{T}$, además otros parámetros relativos a la fisuración, como el Rock Quality Designation (RQD) (3) (4). También se han propuesto modelos que utilizan la caracterización del macizo rocoso, como el RMR (5), o el $\mathrm{Q}_{\mathrm{TBM}}$ (6).

b) Los modelos teóricos se desarrollan a partir de las fuerzas ejercidas sobre los discos cortadores, el equilibrio de fuerzas y el mecanismo de fractura de la roca. Además de los parámetros geomecánicos, precisan datos de la geometría del útil de corte y su disposición en el cabezal, como el diámetro del cortador, el ancho del filo de corte para un determinado desgaste y el espaciamiento entre los filos de corte (1) (7). Bruland (8) además considera el grado de fisuración caracterizado a partir del índice volumétrico de juntas. Roxborough y Phillips (9) tienen en cuenta el ángulo del filo del disco de corte, en vez de su desgaste.

Diversos análisis demostraron que los modelos coinciden cuando se emplean en proyectos donde se conocen bien la resistencia y fisuración de la roca y se miden con precisión los desgates sufridos (1). Sin embargo, cuando se generalizan puede existir bastante divergencia (10) (11), ya que suelen depender de valores medios de las constantes obtenidas mediante métodos estadísticos a partir de los datos de las obras empleadas. Farrokh et al. (12) proponen una forma de suavizar dichas divergencias mediante un nuevo modelo basado en datos de más de $70 \mathrm{~km}$ de túnel en 17 proyectos, donde el diámetro de las galerías va desde los 2,6 a 11 m, con un diámetro más habitual de $3,5 \mathrm{~m}$.

No obstante, la práctica totalidad de los modelos se han desarrollado para tuneladoras, Tunnel Boring Machines (TBM), de diámetro mayor que las microtuneladoras, Micro Tunnel Boring Machines (MTBM), y que emplean discos cortadores más grandes. En consecuencia, los desgastes, los diámetros de los cortadores o la influencia de la relación entre el tamaño de la galería y el espaciamiento de las fisuras serán diferentes.

La MTBM es un sistema de excavación de galerías de diámetro 1.000 a $2.500 \mathrm{~mm}$ que se emplean para la ejecución de túneles de servicio. El sistema realiza el arranque, carga, transporte y sostenimiento de forma continua. Las MTBMs forman parte de las tecnologías sin zanja (13). Según la clasificación de las normas sobre las MTBMs (14), las máquinas empleadas en la presente investigación son máquinas de tipo slurry-shield o hidroescudo sin personal dentro del túnel y guiables. El proceso de corte de las MTBMs coincide con el de las TBMs puesto que se aplica el mismo principio: un dis- co cortador que presiona contra roca en suficiente cantidad como para conseguir arrancar un fragmento. Sin embargo, los discos de corte de las MTBMs son mucho menores, entre $280 \mathrm{~mm}$ (11"), o $305 \mathrm{~mm}$ (12"), frente a los empleados en las TBM, mayores de $356 \mathrm{~mm}$ (14").

Adicionalmente, los proyectos en los que se emplean MTBM, como los analizados en este artículo, tienen las siguientes particularidades frente a los de TBMs:

- Las MTBM instalan tubos prefabricados, que sirven de sostenimiento, y son empujados desde el bastidor, ubicado en el pozo de entrada, por lo que su cometido es, también, transmitir el empuje desde el pozo. Por el contrario, en el ciclo de trabajo de las TBMs se instalan dovelas o sostenimientos convencionales; bulones, gunita, mallazo, etc.

- Las longitudes de los proyectos de MTBM son mucho más cortas, y rara vez superan los $800 \mathrm{~m}$. Las longitudes habituales son menores de $500 \mathrm{~m}$, llegándose incluso a realizar tramos de $50 \mathrm{~m}$ para pasos bajo carreteras o ríos.

- El diámetro de la galería de MTBM, de valor habitual entre 1.000 a $2.500 \mathrm{~mm}$, dificulta las tareas de cambio de los útiles de corte, o los dificulta en exceso por debajo de los $1.200 \mathrm{~mm}$. También es imposible cambiar el cabezal de la máquina a no ser que se excave un pozo y se rescate. Esta maniobra también es complicada dado que muchos de los proyectos se realizan en entorno urbanos.

- Las obras de MTBM se realizan a pequeñas profundidades, habitualmente menores de $20 \mathrm{~m}$, por lo que es frecuente atravesar transiciones entre macizos rocosos sanos y alterados donde la resistencia y grado de fisuración varían notablemente.

- Los proyectos de MTBM tienen una inversión mucho menor que en las TBMs debido a su baja longitud y diámetro. Los limitados recursos económicos no permiten costear un estudio geológico lo suficientemente detallado que requiere la dificultad geológica de perforar en terrenos previsiblemente cambiantes.

Frecuentemente, en obras previstas en macizos muy meteorizados, incluso en suelos, se suele perforar con un cabezal equipado con discos de corte ante la eventual aparición de tramos sanos, debido a la dificultad que supone el cambio de cortadores y el elevado impacto económico que genera ese retraso.

Hegab y Smith (15) analizaron los rendimientos de obras de microtúneles mediante el estudio del tiempo de retraso que tiene lugar únicamente durante la excavación del túnel. A partir de los datos de 35 tramos de distinta longitud y por medio de un tratamiento estadístico, propusieron unas ecuaciones para estimar los tiempos de retraso en las que la única variable que se tiene en cuenta es la longitud del túnel a excavar, no considerando los parámetros geomecánicos de la roca o el resto de operaciones que intervienen en el ciclo de trabajo de una MTBM.

Por tanto, dado que los modelos desarrollados sobre rendimientos de discos de corte fueron obtenidos en proyectos realizados con TBM, este artículo pretende analizar si dichos modelos para predecir los esfuerzos sobre los útiles de corte o estimar el rendimiento del sistema son aplicables a las MTBM y con qué grado de coincidencia.

Para ello se comparan los valores reales de fuerza aplicada sobre los útiles de corte y las velocidades de penetración con los calculados a partir de los modelos propuestos en la literatura 
de TBMs, empleándose los que utilicen datos geomecánicos que se encuentran disponibles en la caracterización geológica del proyecto, principalmente $\sigma_{C}, \sigma_{T}$ y RQD y de geometría de cortadores y presentar un modelo ajustado a las características de las MTBMs.

Además se propone un modelo para estimar las horas totales, $H T$, necesarias para la ejecución de un microtúnel, incluyéndose todas y cada una de las operaciones propias del ciclo de trabajo de la MTBM y no sólo la excavación del microtúnel. Comparados el valor calculado mediante el método y el real se puede obtener un coeficiente de seguridad, $K_{O P}$, o de incertidumbre que indica cuánto podría desviarse el cálculo de la realidad.

El artículo se organiza de la siguiente forma: la sección 2 describe la maquinaria empleada en microtúneles y la medición de los parámetros principales de excavación. En la sección 3 se presenta la base de datos de proyectos de MTBM disponibles y sus características geológicas. En el apartado 4 se comparan los rendimientos reales obtenidos en los tramos analizados con los proporcionados por los modelos existentes sobre TBMs, presentándose un nuevo modelo más ajustado a las MTBMs. Además se presenta un modelo para el cálculo de las horas totales, $H T$, y un sencillo caso práctico. Finalmente, las conclusiones son expuestas en el apartado 5.

\section{DESCRIPCIÓN DE LAS MÁQUINAS MTBM Y PARÁMETROS EMPLEADOS}

Las máquinas empleadas en la ejecución de los microtúneles analizados en este artículo fueron dos máquinas Herrenknecht AVN, cuyas características se presentan en la Tabla 1. Los tubos de diámetros nominales (DN) de $1.600 \mathrm{~mm}$ se instalan en galerías de $2.040 \mathrm{~mm}$ de diámetro de excavación y los de diámetro $2.000 \mathrm{~mm}$ en galerías de $2.475 \mathrm{~mm}$ de diámetro de excavación. Se emplearon cabezales equipados con discos de corte de $280 \mathrm{~mm}$ (11") y $305 \mathrm{~mm}$ (12”).

La máquina MTBM se compone en esencia de dos partes; cuerpo de máquina primero (MC-I) y cuerpo de máquina segundo (MC-II). El MC-I se compone a su vez de dos partes; la parte delantera o móvil y la parte trasera o fija. La parte delantera del MC-I contiene el cabezal perforador y puede orientarse formando un ángulo con respecto a la parte trasera fija, para lo que se precisan al menos 3 cilindros direccionales. Estos cilindros son hidráulicos y tienen una presión máxima de trabajo de 500 bares, siendo la presión que porta cada uno de los cilindros direccionales proporcional a la fuerza que soportan los discos de corte. La presión se mide por medio de un sensor ubicado en el propio cilindro. Variando la carrera de estos cilindros se consigue el guiado de la máquina. La parte trasera de MC-I sirve de apoyo a los cilindros y absorbe los esfuerzos de reacción.

El MC-II porta el grupo electro-hidráulico y la estación remota del autómata que transmite la señal al pupitre de control mediante un cable a lo largo de la galería.

Cuando se empuja desde el bastidor ubicado en el pozo con una fuerza $E$, parte se pierde en el rozamiento entre los tubos y el terreno, $E_{r 2}$, así como en la camisa de la propia máquina, $E_{r 1}$. Al cabezal llega una fuerza $F_{\text {head }}$, calculada mediante la ecuación [1].

$$
F_{\text {head }}=E-E_{r 1}-E_{r 2}
$$

El equipo mide por medio de unos sensores la presión en los cilindros direccionales, por lo que está deducida la fricción desde el puente de empuje hasta el cabezal. Asumiendo como despreciable el rozamiento del perímetro del MC-I con el terreno, que sólo tiene una longitud de 1,5 m, la medida de la presión en los cilindros direccionales proporciona la fuerza total, $F_{\text {head }}$, que soporta el cabezal en cada instante mediante la ecuación [2]:

$$
F_{\text {head }}=\left(p_{1}+p_{2}+p_{3}+p_{4}\right) \cdot S_{\text {cil }}
$$

Donde $p_{1}, p_{2}, p_{3}$ y $p_{4}$ son las presiones medidas en cada uno de los 4 cilindros direccionales; $S_{\text {cil }}$ es la superficie del cilindro, que tiene un valor de $200 \mathrm{~cm}^{2}$ en la máquina AVN160oE y de $250 \mathrm{~cm}^{2}$ en la AVND200o.

En este análisis no se tiene en cuenta el efecto de la posición de los cilindros direccionales, puesto que se operan poco y suele realizarse de forma leve, siendo el máximo ángulo de variación $2,3^{\circ}$ con respecto a la traza inicial. Este ángulo máximo supondría un esfuerzo radial que solamente supone un $4 \%$ de la fuerza total sobre el cabezal, por lo que se considera despreciable.

\begin{tabular}{|c|c|c|}
\hline Modelo & AVN160oE & AVND20oo \\
\hline Fabricante & Herrenknecht & Herrenknecht \\
\hline Diámetro interior nominal del tubo que instala (mm) & 1.600 & 2.000 \\
\hline Diámetro de excavación (mm) & 2.040 & 2.475 \\
\hline Longitud total de la cabeza de corte y de los módulos de potencia (mm) & $7 \cdot 500$ & 10.100 \\
\hline Velocidad de giro del cabezal (rev/min) & $0-8$ & $0-8$ \\
\hline Número de cilindros direccionales & 4 & 4 \\
\hline Carrera de los cilindros direccionales (mm) & 100 & 100 \\
\hline Fuerza que soporta cada cilindro direccional a 500 bar $(\mathrm{kN})$ & 1.000 & 1.250 \\
\hline Potencia motor de corte $(\mathrm{kW})$ & 115 & 315 \\
\hline Par máximo continuo $(\mathrm{m} \cdot \mathrm{kN})$ & 240 & 620 \\
\hline Diámetro cortador (mm) & 280 & 305 \\
\hline Número de discos de corte & 5 simples, 7 dobles & 6 simples, 9 dobles \\
\hline Número de filos (ud) & 19 & 24 \\
\hline Separación entre los filos (mm) & 107 & 103 \\
\hline Longitud de la camisa de la máquina (m) & 7,00 & 10,00 \\
\hline
\end{tabular}

Tabla 1. Características de las máquinas MTBM empleadas. 
El ordenador de la máquina guarda el registro de los parámetros de excavación en intervalos de unos 90 segundos, aunque se pueden programar otros intervalos.

La fuerza por cada filo de corte, $F_{n}$, se obtiene dividiendo $F_{\text {head }}$ por el número de filos de corte del cabezal, $N_{f c}$ (Tabla 1), según la ecuación [3].

$$
F_{n}=\frac{F_{\text {head }}}{N_{f c}}
$$

La penetración por revolución, $P_{r e v}$, se determina a partir del número de revoluciones de la máquina por unidad de tiempo, $w, \mathrm{y}$ la longitud excavada entre dos registros consecutivos, $\Delta l$, registrados en un intervalo de tiempo, $\Delta t$, mediante la ecuación [4]:

$$
P_{r e v}=\frac{\Delta l}{w \cdot \Delta t}
$$

La medición de la longitud $\Delta l$ se realiza mediante un dispositivo denominado coloquialmente «correcaminos», que consta de una rueda que permanece en contacto con el tubo y es capaz de rotar con el avance del mismo, por efecto de la fuerza de rozamiento entre ambos. Además, para poder incrementarla, el dispositivo dispone de un resorte interno que presiona la rueda contra el tubo.

A partir de estos dos parámetros se puede obtener el valor de resistencia a la penetración (field penetration index, FPI), propuesto por Hamilton y Dollinger (16), y que tras ser introducido por Nelson et al. (17), ha sido empleado posteriormente en numerosos estudios sobre excavabilidad con TBM (18). Se trata del cociente entre la fuerza aplicada sobre el cortador y el avance por revolución, que se calcula por medio de la ecuación [5]:

$$
F P I=\frac{F_{n}}{P}
$$

En cada obra se registra, por un lado, el tiempo total, $t_{t}$ (usualmente medido en horas, $H T$ ) empleado en la ejecución del tramo, y, por otro lado, el tiempo neto de perforación, $t_{p}$ (también medido en horas, $H P$ ) cuando el cabezal gira y arranca roca. A partir de estos datos se puede calcular el rendimiento de perforación, rate of penetration, $R O P$, o avance de excavación, que es el ratio entre la longitud del túnel y las horas netas de perforación (ecuación [6]) y la velocidad de avance, $A R$, como ratio entre la longitud del túnel y las horas totales empleadas (ecuación [7]).

$$
\begin{gathered}
R O P=\frac{L}{H P} \\
A R=\frac{L}{H T}
\end{gathered}
$$

Si en la ecuación [4] sustituimos $\Delta l$ por $L$ y $\Delta t$ por $H P$, donde en vez de tomarse un incremento de avance se toma toda la longitud del tramo y el tiempo empleado para su excavación (ecuación [8]), se puede obtener ROP en función de la fuerza aplicada a los útiles de corte, $F_{n}$, y la velocidad de rotación, $w$ (ecuación [10]).

$$
\begin{gathered}
P_{r e v}=\frac{L}{t_{p} \cdot w} \\
R O P=\frac{L}{t_{p}}=P_{r e v} \cdot w \\
R O P=\frac{L}{t_{p}}=\frac{F_{n}}{F P I} \cdot w
\end{gathered}
$$

\begin{tabular}{|c|c|c|c|c|c|c|c|}
\hline Proyecto: Promotor & $\begin{array}{c}\text { Ensayos } \\
\text { disponibles }\end{array}$ & Tramo & $\begin{array}{c}\mathrm{DN} \\
(\mathrm{mm})\end{array}$ & $\begin{array}{c}\mathbf{L} \\
(\mathbf{m})\end{array}$ & $\begin{array}{c}\text { Dc } \\
(\mathbf{m m})\end{array}$ & Litología & GM \\
\hline \multirow{3}{*}{$\begin{array}{l}\text { Interceptor del Nervión, tramo Arrigorriaga-San Miguel: } \\
\text { Consorcio de Aguas de Bilbao }\end{array}$} & \multirow{3}{*}{$\begin{array}{l}6 \text { UCS } \\
1 \text { BTS }\end{array}$} & ARR-1 & 1.600 & 377,80 & 280 & Marga & II \\
\hline & & ARR-2 & 1.600 & 493,40 & 280 & Marga & II \\
\hline & & ARR-3 & 1.600 & 451,60 & 280 & Marga & II \\
\hline \multirow{4}{*}{$\begin{array}{l}\text { Interconexión de redes de abastecimiento del Maresme } \\
\text { Nord Tramo 3: ATLL }\end{array}$} & \multirow{4}{*}{$\begin{array}{l}23 \text { UCS } \\
3 \text { BTS }\end{array}$} & CAL-1 & 1.600 & 218,18 & 280 & Granodiorita & IV \\
\hline & & CAL-2 & 1.600 & 287,33 & 280 & Granodiorita & II \\
\hline & & CAL-3 & 1.600 & 212,96 & 280 & Granodiorita & IV \\
\hline & & CAL-4 & 1.600 & 328,40 & 280 & Granodiorita & IV \\
\hline \multirow{2}{*}{$\begin{array}{l}\text { Colectores del río Miño (Lugo). Tramo VI-EDAR: } \\
\text { Confederación Hidrográfica del Norte }\end{array}$} & \multirow{2}{*}{$\begin{array}{l}24 \text { UCS } \\
4 \text { BTS }\end{array}$} & LUG-1 & 1.600 & 192,89 & 280 & Esquisto & II \\
\hline & & LUG-2 & 1.600 & 359,63 & 280 & Esquisto & II \\
\hline Colector del Urumea: Consorcio de Aguas de Añarbe & $\begin{array}{l}1 \text { UCS } \\
1 \text { BTS }\end{array}$ & URU-1 & 1.600 & 78,40 & 280 & Marga & II \\
\hline Emisario Mompas: Confederación Hidrográfica del Norte & $\begin{array}{l}1 \text { UCS } \\
5 \text { BTS }\end{array}$ & MOM-1 & 2.000 & 100,88 & 305 & Flysh & II \\
\hline $\begin{array}{l}\text { Regenüberlaufstauraum-kanal Rüsk: Ayuntamiento } \\
\text { de Nuremberg }\end{array}$ & $\begin{array}{l}2 \text { UCS } \\
1 \text { BTS }\end{array}$ & NUR-2 & 2.000 & 99,30 & 305 & Calcarenita & IV \\
\hline \multirow{2}{*}{$\begin{array}{l}\text { Ampliación del colector C-2 de Pamplona: } \\
\text { Mancomunidad de Aguas de Pamplona }\end{array}$} & \multirow{2}{*}{$\begin{array}{l}26 \text { UCS } \\
1 \text { BTS }\end{array}$} & PAM-1 & 2.000 & 305,41 & 305 & Marga & II \\
\hline & & PAM-3 & 2.000 & 386,47 & 305 & Marga & II \\
\hline $\begin{array}{l}\text { Adecuación del entorno del paseo fluvial del río Cares en } \\
\text { Panes (Asturias): Confederación Hidrográfica del Norte }\end{array}$ & $\begin{array}{l}3 \text { UCS } \\
1 \text { BTS }\end{array}$ & PAN-1 & 2.000 & 419,93 & 305 & Marga & II \\
\hline
\end{tabular}

\section{PROYECTOS ANALIZADOS Y GEOLOGÍA}

La base de datos empleada en este artículo se obtuvo a partir de los valores registrados durante la ejecución de 15 tramos de microtúneles en 8 proyectos de 2002 a 2010, sumando un total de $4,5 \mathrm{~km}$, con tubos de diámetro interior de $1.600 \mathrm{y}$ $2.000 \mathrm{~mm}$ (Tabla 2).

Tabla 2. Características de los tramos de microtúnel utilizados en el estudio.

Notas. DN: diámetro nominal del tubo, L: longitud del tramo, Dc: diámetro del disco de corte, GM: grado de meteorización de la roca. 
CAL-2

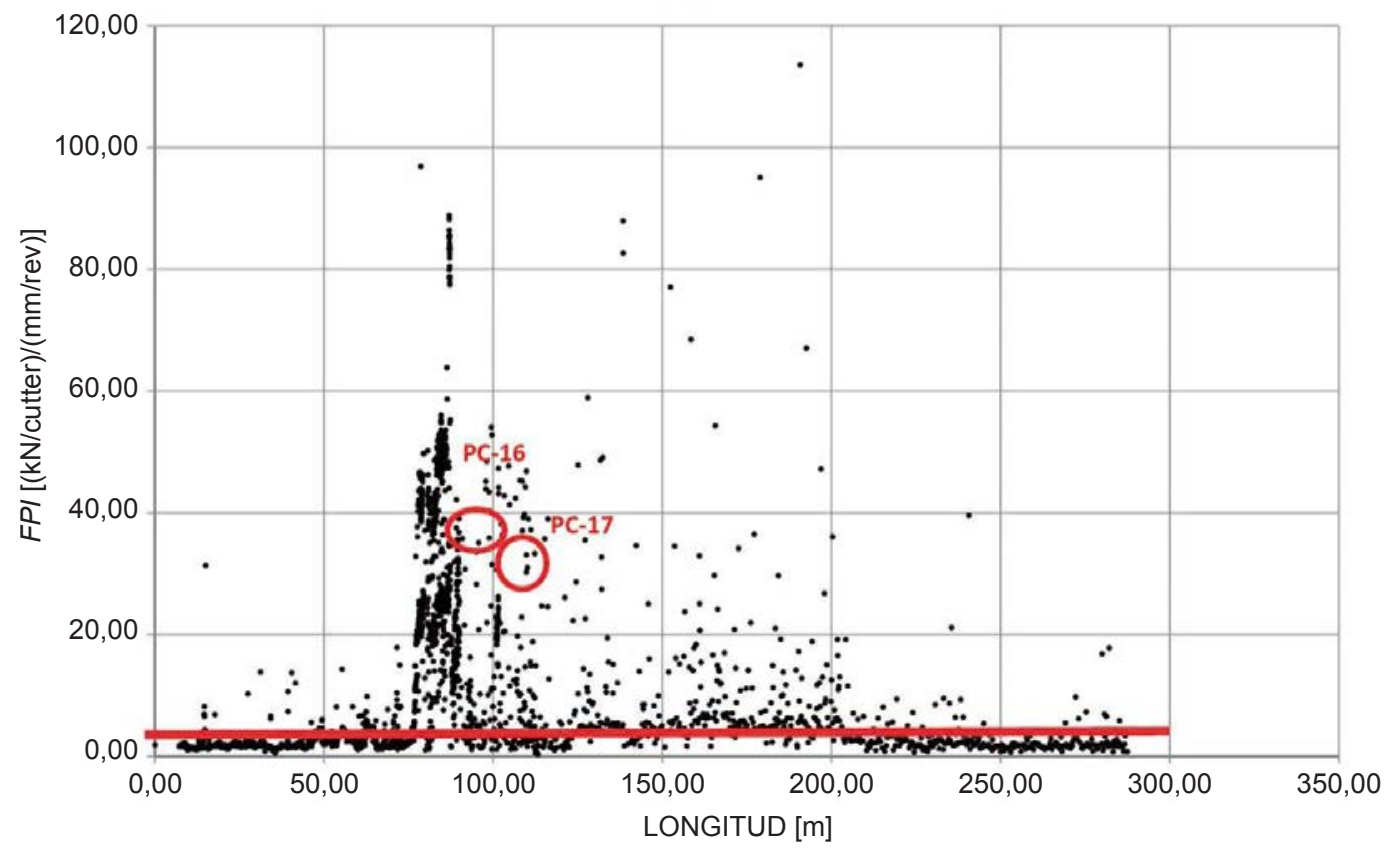

Figura 1. Valores de FPI obtenidos en el tramo CAL-2 cada 90 segundos de perforación.

La profundidad media de la excavación fue inferior a $20 \mathrm{~m}$ excepto en LUG-1 donde se alcanzaron hasta $100 \mathrm{~m}$ de profundidad. Las características mecánicas de los macizos rocosos atravesados se conocen a partir de los estudios geológicos y geotécnicos de cada proyecto, en los que se incluyen sondeos en la traza del túnel y fuera de ella. Existe una gran variabilidad en relación a la cantidad de ensayos disponibles.

\section{RESULTADOS Y DISCUSIÓN}

\subsection{Correlación entre los valores obtenidos mediante los modelos existentes y los reales}

Para cada uno de los 15 tramos de túnel analizados se obtuvo un diagrama como el de la Figura 1 (correspondiente a CAL-2), donde se muestra la medición de FPI obtenida en cada intervalo de tiempo, generalmente de 90 segundos, mediante un punto, a lo largo de la longitud de cada tramo. La línea horizontal indica el valor medio de FPI observado de cada tramo. La Tabla 3 recoge los valores medios de rendimiento de excavación $\left(F_{n}, P_{r e v}, w, F P I\right)$ y de características geomecánicas de cada tramo. El valor medio de FPI no coincide con el ratio entre los valores de $F_{n}$ y $P_{\text {rev }}$ presentados en la Tabla 3 dado que estos últimos se corresponden con el promedio de valores registrados a lo largo de todo el tramo. La caracterización geomecánica de cada tramo se llevó a cabo por medio de los ensayos disponibles. En caso de atravesarse varias litologías, se calcularon los valores proporcionalmente a la longitud excavada en cada litología, según los perfiles geológicos disponibles.

Además, en la Figura 1, rodeados en círculos, se indican los puntos donde el trazado del túnel atraviesa el lugar donde se había realizado un sondeo, denominados puntos de control, PC. De esos puntos concretos se conocen las características geomecánicas a través de los sondeos que existían en el proyecto, así como los rendimientos de excavación $\left(F P I, w, F_{n}\right.$ y $\left.P_{\text {rev }}\right)$

Tabla 3. Valores medios de los parámetros principales en cada tramo analizado.

\begin{tabular}{|c|c|c|c|c|c|c|c|c|}
\hline Tramo & $\begin{array}{l}F_{\text {head }} \\
(\mathbf{k N})\end{array}$ & $\begin{array}{c}F_{n} \\
(\mathbf{k N})\end{array}$ & $\begin{array}{c}w \\
\text { (rev/min) }\end{array}$ & $\begin{array}{c}P_{\text {rev }} \\
(\mathrm{mm} / \mathrm{rev})\end{array}$ & $\begin{array}{c}F P I \\
(\mathrm{kN} / \mathrm{mm})\end{array}$ & $\begin{array}{c}\sigma_{c} \\
(\mathbf{M P a})\end{array}$ & $\begin{array}{c}\sigma_{T} \\
(\mathbf{M P a})\end{array}$ & $\begin{array}{c}\text { RQD } \\
(\%)\end{array}$ \\
\hline ARR-1 & 936 & 49,3 & 6,2 & 5,1 & 12,8 & 34,46 & 4,11 & 75,00 \\
\hline ARR-2 & 805 & 42,3 & 4,4 & 7,7 & 8,7 & 27,09 & 4,32 & 100,00 \\
\hline ARR-3 & 890 & 46,8 & 4,1 & 6,2 & 9,9 & 19,73 & 4,53 & 80,00 \\
\hline CAL-1 & 756 & 39,9 & 4,1 & 12,8 & 3,9 & 3,60 & 1,28 & 0,00 \\
\hline CAL-2 & 925 & 48,7 & 4,2 & 10,0 & 13,3 & 33,96 & 3,46 & 25,90 \\
\hline CAL-3 & 519 & 27,3 & 4,3 & 11,1 & 3,0 & 24,12 & 2,76 & 15,00 \\
\hline CAL-4 & 797 & 42,0 & 4,2 & 11,4 & 5,3 & 34,78 & 3,52 & 22,80 \\
\hline LUG-1 & 957 & 50,3 & 7,9 & 2,0 & 38,4 & 54,17 & 10,06 & 58,33 \\
\hline LUG-2 & 1.104 & 58,1 & 7,5 & 1,8 & 34,4 & 73,63 & 9,22 & 58,33 \\
\hline URU-1 & 825 & 43,5 & 6,1 & 5,8 & 12,9 & 50,60 & 7,62 & 80,00 \\
\hline MOM-1 & 1.482 & 61,8 & 3,6 & 7,6 & 11,2 & 60,03 & 7,00 & 60,00 \\
\hline NUR-2 & 1.889 & 78,7 & 4,1 & 9,6 & 10,9 & 25,00 & 2,00 & 90,00 \\
\hline PAM-1 & 2.209 & 92,1 & 3,4 & 13,6 & 7,3 & 13,15 & 6,80 & 60,00 \\
\hline PAM-3 & 1.930 & 80,4 & 3,6 & 10,8 & 7,1 & 10,50 & 5,26 & 90,00 \\
\hline PAN-1 & 1.770 & 73,5 & 3,7 & 8,5 & 12,2 & 17,85 & 0,07 & 65,00 \\
\hline
\end{tabular}


en el entorno de ese punto, $5 \mathrm{~m}$ antes y $5 \mathrm{~m}$ después del lugar del sondeo (Tabla 4). De esta forma, tanto los valores medios de rendimiento de la excavación ( $F P I, F_{n}$ y $P_{\text {rev }}$ ) de todo el tramo y las características geomecánicas medias del tramo (Tabla 3), como los valores puntuales de rendimiento en el entorno y las características geomecánicas de los PC (Tabla 4) se utiliza- ron para la comprobación de los valores obtenidos mediante las fórmulas existentes sobre rendimiento de TBMs.

Las fórmulas propuestas en los principales modelos desarrollados para la predicción del rendimiento de TBM, tanto empíricas como teóricas, se muestran en la Tabla 5.

Tabla 4. Valores de los parámetros principales en el entorno de los sondeos, puntos de control.

\begin{tabular}{|c|c|c|c|c|c|c|c|c|}
\hline $\begin{array}{c}\text { Punto de } \\
\text { control }\end{array}$ & Tramo & $\begin{array}{c}F_{n} \\
(\mathbf{k N})\end{array}$ & $\begin{array}{c}w \\
\text { (rev/min) }\end{array}$ & $\begin{array}{c}P_{r e v} \\
(\mathrm{~mm} / \mathrm{rev})\end{array}$ & $\begin{array}{c}F P I \\
(\mathrm{kN} / \mathrm{mm})\end{array}$ & $\begin{array}{c}\sigma_{c} \\
(\mathbf{M P a})\end{array}$ & $\begin{array}{c}\sigma_{T} \\
(\mathbf{M P a})\end{array}$ & $\begin{array}{c}\text { RQD } \\
(\%)\end{array}$ \\
\hline PC-2 & ARR-1 & 38,20 & 4,5 & 5,14 & 11,06 & 7,48 & 4,81 & 100,00 \\
\hline $\mathrm{PC}-3$ & ARR-2 & 28,20 & 4,5 & 5,40 & 7,56 & 2,88 & 4,81 & 100,00 \\
\hline PC-5 & ARR-2 & 54,12 & 4,5 & 3,72 & 19,90 & 76,30 & 3,42 & 100,00 \\
\hline PC-6 & ARR-3 & 48,35 & 2,19 & 11,97 & 5,87 & 13,29 & 4,81 & 80,00 \\
\hline $\mathrm{PC}-16$ & CAL-2 & 65,51 & 4,2 & 2,70 & 33,56 & 80,76 & 7,18 & 69,33 \\
\hline $\mathrm{PC}-17$ & CAL-2 & 60,18 & 4,42 & 2,67 & 27,01 & 75,00 & 7,18 & 69,33 \\
\hline PC-18 & CAL-3 & 30,42 & 4,24 & 10,14 & 3,10 & 6,19 & 1,28 & 60,00 \\
\hline PC-20 & CAL-4 & 65,64 & 4,2 & 4,88 & 16,81 & 30,36 & 4,23 & 60,00 \\
\hline $\mathrm{PC}-35$ & LUG-2 & 56,99 & 7,1 & 1,80 & 33,29 & 57,70 & 6,46 & 75,00 \\
\hline $\mathrm{PC}-36$ & LUG-2 & 65,04 & 7,2 & 1,66 & 42,04 & 99,20 & 10,08 & 50,00 \\
\hline $\mathrm{PC}-37$ & LUG-2 & 67,80 & 7,1 & 1,71 & 39,97 & 126,13 & 10,03 & 50,00 \\
\hline $\mathrm{PC}-42$ & MOM-1 & 65,93 & 3,65 & 6,07 & 14,04 & 60,80 & 7,08 & 60,00 \\
\hline PC-45 & PAM-1 & 97,51 & 3,4 & 11,56 & 9,41 & 13,10 & 7,92 & 60,00 \\
\hline PC-47 & PAM-1 & 81,30 & 3,4 & 8,65 & 11,78 & 19,20 & 5,67 & 60,00 \\
\hline $\mathrm{PC}-48$ & PAM-3 & 77,98 & 3,7 & 11,16 & 7,00 & 11,20 & 5,67 & 90,00 \\
\hline PC-49 & PAM-3 & 78,65 & 3,72 & 10,71 & 7,46 & 10,10 & 4,46 & 90,00 \\
\hline PC-52 & PAM- 3 & 72,21 & 3,45 & 9,52 & 8,33 & 12,35 & 5,67 & 90,00 \\
\hline PC-53 & PAN-1 & 66,92 & 3,5 & 8,95 & 14,72 & 16,94 & 0,06 & 65,00 \\
\hline PC-54 & PAN-1 & 61,78 & 3,8 & 7,82 & 8,30 & 18,78 & 0,08 & 65,00 \\
\hline
\end{tabular}

Tabla 5. Principales modelos de rendimiento de tuneladoras (TBM).

\begin{tabular}{|c|c|c|}
\hline Autor/es (año) & Fórmula propuesta & Ecuación \\
\hline Roxborough y Phillips (1975) (9) & $F_{n}=4 \cdot \sigma_{C} \cdot \tan (\phi / 2) \cdot \sqrt{D \cdot P^{3}-P^{4}}$ & [11] \\
\hline Graham (1976) (19) & $P_{r e v}=\frac{3 \cdot 940 \cdot F_{n}}{\sigma_{C}}$ & [12] \\
\hline Farmer y Glossop (1980) (20) & $P_{r e v}=\frac{624 \cdot F_{n}}{\sigma_{T}}$ & [13] \\
\hline Cassinelli et al. (1982) (21) & $P_{r e v}=-0,0059 \cdot R S R+1,59$ & [14] \\
\hline Innaurato et al. (1991) (22) & $P_{r e v}=40,41 \cdot \sigma_{C}^{-0,44}+0,047 \cdot R S R+3,15$ & [15] \\
\hline Rostami (1997) (23) & $F_{t}=\frac{P^{o} \cdot \Phi \cdot R \cdot T}{1+\psi} ; \quad \Phi=\cos ^{-1}\left(\frac{R-P_{r e v}}{R}\right) ; \quad P^{o}=C \cdot \sqrt[3]{\frac{S \cdot \sigma_{C}^{2} \cdot \sigma_{T}}{\Phi \cdot \sqrt{R \cdot T}}}$ & {$[16][17][18]$} \\
\hline Yaguiz (2008) (24) & $R O P=1,093+0,029 P S I-0,003 \sigma_{C}+0,437 \log (\alpha)-0,219 D P W$ & [19] \\
\hline Gong y Zhao (2009) (25) & $B I_{(1)}=37,06 \cdot \sigma_{C}^{0,26} \cdot B i^{-0,1} \cdot\left(0,84 \cdot e^{-0,05 J_{V}}+e^{-0,09 \cdot \sin (\alpha+30)}\right)$ & [20] \\
\hline Hassanpour et al. (2009) (3) & $F P I=\exp \left(0,004 \cdot \sigma_{C}+0,008 \cdot R Q D+2,077\right)$ & [21] \\
\hline Khademi Hamidi et al. (2010) (26) & $F P I=4,161+0,091 \sigma_{C}+0,077 R Q D+0,117 J_{C}+1,077 \log (\alpha)$ & [22] \\
\hline Hassanpour et al. (2011) (27) & $F P I=\exp \left(0,015 \cdot \sigma_{C}+0,008 \cdot R Q D+1,384\right)$ & [23] \\
\hline Farrokh et al. (2012) (12) & $\begin{array}{l}P_{r e v}=\exp \left(0,41+0,404 \cdot D_{t}-0,027 \cdot D_{t}^{2}+0,0691 \cdot R T_{C}-0,00431 \cdot \sigma_{C}+\right. \\
\left.+0,0902 \cdot R Q D_{C}+0,000893 \cdot F_{n}\right)\end{array}$ & {$[24]$} \\
\hline
\end{tabular}

Notas. ø/2: medio ángulo del filo del cortador (rad); $D$ : diámetro del cortador; $P$ : penetración (mm); RSR: rock structure rating; $F$ : fuerza total resultante, $T$ : ancho del filo de corte; $R$ : radio del cortador; $P^{\circ}$ : presión base en la zona fracturada; $\psi$ : constante de la función de distribución de la presión, habitualmente entre -0,2 y 0,2; $C$ : constante, de valor aproximado 2,12; $S$ : espaciado entre cortadores; $J_{c}$ : valor de la condición de las juntas en el sistema RMR; $P S I$ : peak slope index en el test Punch; $\alpha$ : ángulo entre el eje del túnel y el plano de juntas; $D P W$ : distancia entre planos de debilidad, según Bruland (1999); $B I$ : specific rock mass boreability index (índice de excavabilidad específico del macizo rocoso); Bi: índice de fragilidad de la roca; $J_{v}$ : recuento volumétrico de juntas; $D_{t}$ : diámetro del túnel (m); $R T_{c}$ : código del tipo de roca; $R Q D_{c}:$ código $R Q D$. 
De los modelos presentados en la Tabla 5 no todos pueden ser utilizados en proyectos de microtuneladoras, dado que no están disponibles todos los ensayos que cada modelo necesita. Se evalúan los valores obtenidos mediante las fórmulas de Roxborough y Phillips (9), Graham (19), Farmer y Glossop (20), Rostami (23) y Hassanpour et al. (3), aplicada esta última para rocas carbonato-arcillosas, como las presentes en los tramos analizados. Los coeficientes de determinación $R^{2}$ obtenidos entre los modelos propuestos y los valores obtenidos se muestran en la Tabla 6.

A la vista de los valores obtenidos, se estimó que dichas fórmulas no eran válidas para el uso en microtuneladoras, por lo que, utilizando los datos disponibles, se examinó la relación entre los parámetros geológicos de cada tramo y de los puntos de control (PC) con los parámetros de rendimiento de la MTBM, mediante análisis de regresión lineal simple (Tabla 7).

Como se observa, el FPI muestra una mejor correlación con los parámetros geológicos y, por consiguiente, fue seleccionado para desarrollar una relación empírica con los parámetros geológicos y de la máquina mediante una regresión lineal múltiple. Se utilizaron como variables independientes (o predictores) la resistencia a compresión simple, $\sigma_{c}$; la resistencia a tracción, $\sigma_{\mathrm{T}}$; el Rock Quality Designation, $R Q D$; el diámetro de los discos, $D$, y la separación entre discos, $S$. La influencia de cada variable en el FPI fue evaluada usando un análisis de regresión por pasos hacia adelante mediante el programa SPSS v24.0. Tras estudiar distintas combinaciones la mejor correlación se encontró entre el parámetro $\ln (F P I)$ y las variables independientes $U C S$ y $R Q D$. La ecuación obtenida es:

$$
F P I=\exp \left(0,0193 \cdot \sigma_{C}+0,007 \cdot R Q D+1,303\right)
$$

Con FPI expresado en (kN/cutter)/(mm/rev), $R Q D$ en porcentaje (\%) y $\sigma_{C}$ en MPa. El coeficiente de determinación $\left(R^{2}\right)$ es 0,697 y el coeficiente $R^{2}$ ajustado es 0,678 , indicando que la regresión planteada explica el $70 \%$ de la variabilidad de los datos disponibles. La prueba de significación indica que existe una relación lineal significativa entre las variables. Otros supuestos que se deben cumplir en los análisis de regresión lineal múltiple son la independencia, la homocedasticidad y la normalidad de los residuos y la no colinealidad. La independencia de los residuos se puede evaluar con el estadístico de Durbin-Watson (DW), cuyo valor varía entre o y 4, asu- miéndose que hay independencia con valores entre 1,5 y 2,5, y se obtiene un valor de 1,969. La normalidad de residuos se comprueba en la Figura 2a) donde se observa cómo los residuos estandarizados (o tipificados) se ajustan a la recta de distribución normal. La homocedasticidad se comprueba en la Figura 2b) donde se observa que la variación de los residuos no sigue ningún patrón. La no colinealidad queda demostrada con un nivel de tolerancia 0,983, superior a 0,10 y un valor de FIV de 1,108, no superior a 10. Además, un análisis de la $F$ de Snedecor y de la $t$ de Student de los resultados indica que la correlación es real $(\mathrm{p}<0,05)$ y que los de regresión son verdaderos, o sea, significativamente distintos de cero $(\mathrm{p}<0,05)$.

\subsection{Modelo para la predicción de las horas totales de trabajo en un microtúnel}

Los proyectos que emplean MTBM se caracterizan por ser de corta longitud, y en consecuencia de corta duración, oscilando los plazos entre 2 o 3 semanas hasta 3 o 4 meses. Por tanto, en una actividad tan dinámica resulta útil disponer de un modelo que permita determinar el tiempo de ejecución del tramo desde que se inicia el montaje hasta que se retira el equipo para ser trasladado a otro lugar, teniendo en cuenta el ciclo de trabajo de las MTBMs.

La cantidad de horas totales, HT(h), necesarias para la ejecución de un tramo de longitud, $L(\mathrm{~m})$, incluyendo los tiempos en los que la máquina no excava se puede determinar mediante la ecuación [29]

$$
\begin{gathered}
H T=K_{O P} \cdot\left(\frac{L}{R O P}+\frac{L}{L_{T}} \cdot T_{N T}+\frac{L}{L_{T}} \cdot T_{M R P}+\frac{L}{L_{C}} \cdot T_{M C}+\right. \\
\left.+\frac{L}{L_{A G}} \cdot T_{M A G}+\frac{L}{L_{T O P}} \cdot T_{M T O P}+\frac{L}{L_{M A N}} \cdot T_{M M A N}+T I\right)
\end{gathered}
$$

El significado de las variables empleadas en la ecuación [29] se presenta en la Tabla 8, donde además se expone a qué parte del ciclo de trabajo de la MTBM pertenece cada variable.

Se ponen de manifiesto las diferencias entre el ciclo de excavación de las MTBM y el que propone Bruland (8) para las TBM.

Tabla 6. Coeficientes de determinación para las fórmulas de rendimiento de TBMs analizadas con los datos reales.

\begin{tabular}{|l|c|c|c|}
\hline \multicolumn{1}{|c|}{ Modelo } & Ecuación & Parámetro estimado & Coef. determinación ( $\left.\boldsymbol{R}^{2}\right)$ \\
\hline Roxborough y Phillips (1975) (9) & {$[11]$} & $F_{n}$ & 0,0035 \\
\hline Graham (1976) (19) & {$[12]$} & $P_{\text {rev }}$ & 0,3774 \\
\hline Farmer y Glossop (1980) (20) & {$[13]$} & $P_{\text {rev }}$ & 0,012 \\
\hline Rostami et al. (1997) (23) & {$[16][17][18]$} & $F_{n}$ & 0,009 \\
\hline Hassanpour et al. (2009) (3) & {$[21]$} & $F P I$ & 0,20 \\
\hline
\end{tabular}

Tabla 7. Resumen de los resultados del análisis estadístico y coeficientes de determinación $\left(R^{2}\right)$.

\begin{tabular}{|c|c|c|c|c|c|c|}
\hline \multirow{2}{*}{ Parámetro } & \multicolumn{3}{|c|}{ Coeficiente de determinación (R2) } & \multirow{2}{*}{ Correlación } & \multirow{2}{*}{ Eq. n. } \\
\cline { 2 - 7 } & ROP & $\mathbf{P}_{\text {rev }}$ & FPI & $\begin{array}{c}\text { Tipo } \\
\text { relación }\end{array}$ & $F P I=0,3178 \cdot \sigma_{C}+3,6667$ & {$[25]$} \\
\hline$\sigma_{C}$ & 0,614 & 0,667 & 0,725 & Lineal & $F P I=2,6864 \cdot \sigma_{T}+1,4115$ & {$[26]$} \\
\hline$\sigma_{T}$ & 0,336 & 0,378 & 0,436 & Lineal & $R O P=0,0004 \cdot R Q D^{2}-0,0527 \cdot R Q D+3,365$ & {$[27]$} \\
\hline$R Q D$ & 0,273 & 0,142 & 0,187 & Polinómica & &
\end{tabular}


a)

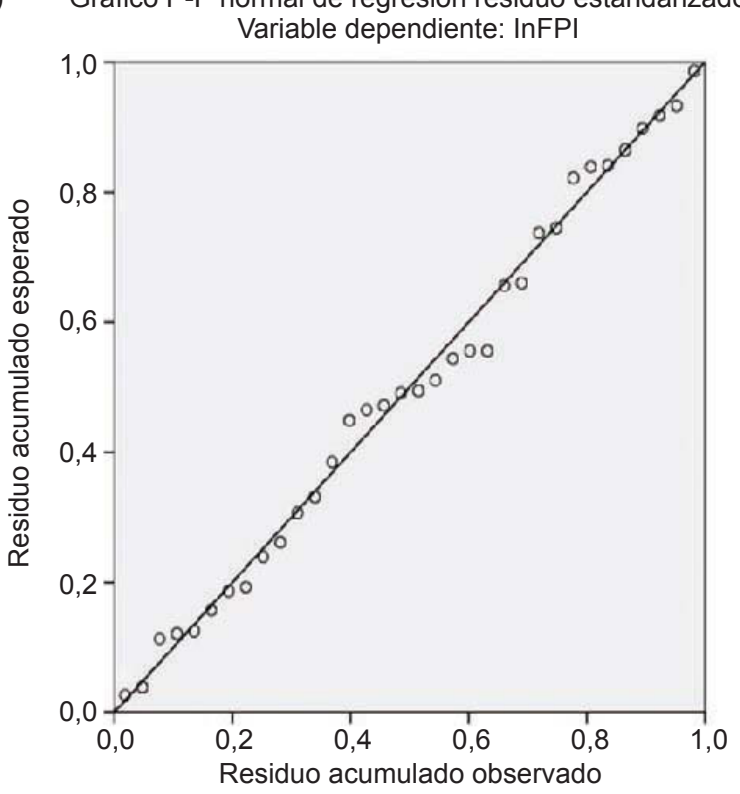

b)

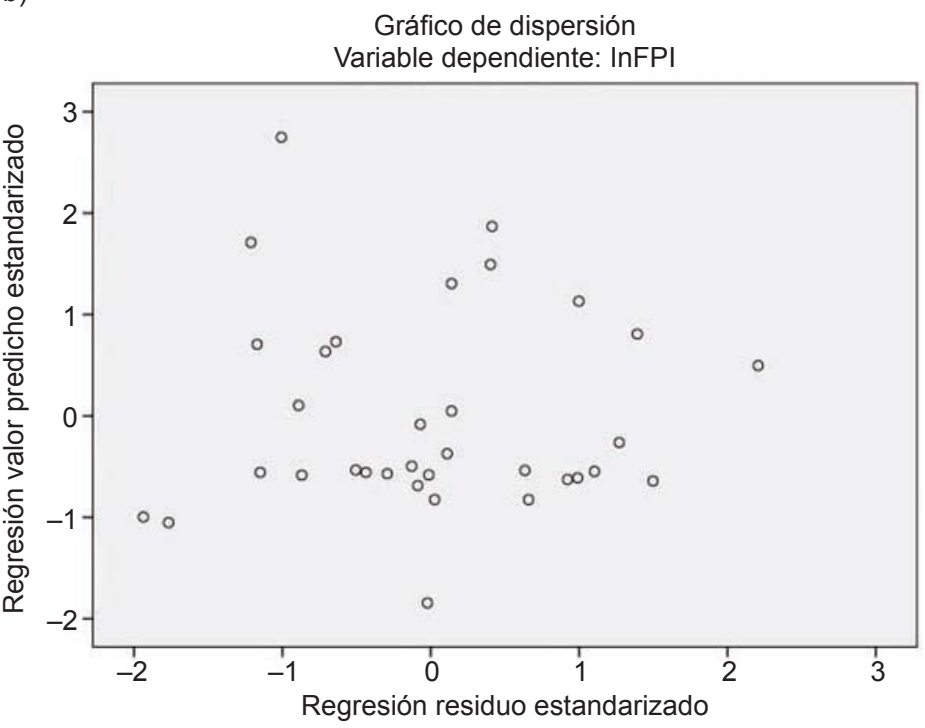

Figura 2. a) Gráfico normal de regresión del residuo estandarizado. b) Gráfica de dispersión de los residuos estandarizados.

Con respecto a las variables descritas y a sus valores habituales expuestos se realizan las siguientes indicaciones:

- La operación de cambio de discos de corte en microtuneladoras es compleja debida a la falta de espacio. Un valor de $T_{M C}$ de 24 a $36 \mathrm{~h}$ de trabajo puede resultar bajo en caso de que surgieran problemas.

- La vida de los cortadores, $L_{c}$, depende de la abrasividad y de la fuerza aplicada sobre los mismos. En las obras analizadas en este artículo la vida de los cortadores ha variado entre 70 y $450 \mathrm{~m}$. Generalmente las obras se ejecutan sin cambiar los útiles de corte, bien por ser cortos los tramos, bien por la baja abrasividad del terreno o bien porque se aprovechó al máximo la vida útil de los discos para evitar un costoso cambio de cortadores. En los casos analizados únicamente se efectuaron cambios de discos en los tramos LUG-2, CAL-1 y CAL-2. Al final de cada obra se comprobó el desgaste de los cortadores, el cual era muy variable. Resulta inviable medir el desgaste con mayor frecuencia debido a la dificultad y coste que supone el acceso al frente.

- La longitud que se puede excavar sin cambiar el agua del tanque, $L_{A G}$, depende de la cantidad de finos que se pro-

Tabla 8. Ciclo de trabajo de la MTBM y significado de las variables empleadas en la ecuación [29].

\begin{tabular}{|c|c|c|c|}
\hline $\begin{array}{l}\text { Fase del ciclo } \\
\text { de trabajo }\end{array}$ & Variable utilizada & $\begin{array}{l}\text { Unidades } \\
\text { empleadas }\end{array}$ & Valores habituales \\
\hline Perforación & $R O P$ : rendimiento de perforación. & $\mathrm{m} / \mathrm{h}$ & Distinto en cada proyecto \\
\hline \multirow{2}{*}{$\begin{array}{l}\text { Colocación de tubo } \\
\text { nuevo }\end{array}$} & $L_{T}:$ Longitud del tubo & $\mathrm{m}$ & De 2,4 a $3,0 \mathrm{~m}$ \\
\hline & $T_{N T}:$ Tiempo habitual de instalación de nuevo tubo & $\mathrm{h}$ & De 0,5 a $0,7 \mathrm{~h}$ \\
\hline \multirow{2}{*}{$\begin{array}{l}\text { Tiempo de } \\
\text { reanudación } \\
\text { de la perforación }\end{array}$} & $L_{T}:$ Longitud del tubo & $\mathrm{m}$ & De 2,4 a $3,0 \mathrm{~m}$ \\
\hline & $\begin{array}{l}T_{M R P}: \text { Tiempo medio de reanudación de la perforación tras cada tubo } \\
\text { nuevo (alinear láser, cebar bombas, etc.) }\end{array}$ & $\mathrm{h}$ & De 0,09 a $0,25 \mathrm{~h}$ \\
\hline \multirow{2}{*}{$\begin{array}{l}\text { Cambio de } \\
\text { cortadores }\end{array}$} & $L_{c}:$ Longitud excavable con el mismo cortador, vida de los cortadores & $\mathrm{m}$ & $\begin{array}{l}\text { Variable según cortador } \\
\text { y terreno a atravesar }\end{array}$ \\
\hline & $T_{M C}:$ Tiempo medio de cambio de cortadores & $\mathrm{h}$ & De 24 a $36 \mathrm{~h}$ \\
\hline \multirow{2}{*}{$\begin{array}{l}\text { Cambio de agua } \\
\text { de perforación }\end{array}$} & $L_{A G}:$ Longitud que se puede perforar sin cambiar el agua del tanque & $\mathrm{m}$ & De 10 a $100 \mathrm{~m}$ \\
\hline & $T_{M A G}:$ Tiempo medio de cambio de agua del tanque & $\mathrm{h}$ & $2 \mathrm{~h}$ \\
\hline \multirow{2}{*}{$\begin{array}{l}\text { Comprobación } \\
\text { topográfica del } \\
\text { trazado }\end{array}$} & $\begin{array}{l}L_{T O P}: \text { Longitud que se puede perforar entre dos comprobaciones } \\
\text { topográficas }\end{array}$ & $\mathrm{m}$ & $20 \mathrm{~m}$ \\
\hline & $T_{M T O P}:$ Tiempo empleado en realizar la comprobación topográfica & $\mathrm{h}$ & De $1 \mathrm{a} 2 \mathrm{~h}$ \\
\hline \multirow{2}{*}{$\begin{array}{l}\text { Mantenimiento } \\
\text { del equipo }\end{array}$} & $\begin{array}{l}L_{\text {MAN }}: \text { Longitud que se puede perforar sin realizar parada de } \\
\text { mantenimiento preventivo del equipo }\end{array}$ & $\mathrm{m}$ & $100 \mathrm{~m}$ \\
\hline & $\begin{array}{l}T_{M M A N}: \text { Tiempo medio empleado para el mantenimiento preventivo } \\
\text { del equipo }\end{array}$ & $\mathrm{h}$ & $6 \mathrm{~h}$ \\
\hline Imprevistos & $\begin{array}{l}\text { TI: Tiempo asignado a imprevistos que se puedan conocer con } \\
\text { anterioridad. Si se hace uso del coeficiente } K_{O P} \text {, asignar } T I=0\end{array}$ & $\mathrm{~h}$ & Variable \\
\hline $\begin{array}{l}\text { Rendimiento } \\
\text { del ciclo }\end{array}$ & $\begin{array}{l}K_{O P}: \text { rendimiento global que tiene en cuenta incertidumbres } \\
\text { geológicas y debidas a otras causas }\end{array}$ & - & De 1 a 5 \\
\hline
\end{tabular}


duzcan durante el arranque y trituración de los fragmentos (chips) en la cámara, así como de los medios disponibles en la planta de separación. En excavaciones en roca puede variar entre los $10 \mathrm{~m}$ para rocas que generan muchos finos (margas, lutitas, esquistos) a más de $50 \mathrm{~m}$ en granitos. No realizar el cambio de agua de perforación con regularidad puede reducir el rendimiento de perforación.

- La topografía para la comprobación de la alineación de la máquina debe realizarse sin empuje de los tubos y, por lo tanto, implica una parada. Además, a partir de los $100 \mathrm{~m}$ de longitud puede ser necesario realizar estaciones dentro del tubo, lo que implica una ralentización del proceso debido a las limitaciones de espacio.

- Una vez calculado el FPI mediante la ecuación [28] para estimar el $R O P$ sólo es necesario introducir los valores de $F_{n}$ y rpm de las máquinas disponibles en la ecuación [10]. Los valores habituales de rpm varían entre 4 y $8 \mathrm{rev} / \mathrm{min}$, mientras que el valor de $F_{n}$ dependerá de la microtuneladora escogida. Para un estudio preliminar se propone la ecuación [30] que mediante un análisis de regresión lineal múltiple, similar al llevado a cabo para predecir FPI, relaciona $R O P$ con los mismos parámetros utilizados para el FPI.

$$
R O P=3,289-0,019 \cdot \sigma_{C}-0,012 \cdot R Q D
$$

Con $R O P$ expresado en $\mathrm{m} / \mathrm{h}, R Q D$ en porcentaje (\%) y $\sigma_{C}$ en MPa. El coeficiente $R^{2}$ obtenido es 0,678 y el $R^{2}$ ajustado es 0,658 . Se cumplen igualmente la independencia (DW = 1,509), la homocedasticidad y la normalidad de residuos y la no colinealidad (nivel de tolerancia $=0,983$ y FIV $=1,018$ ). $\mathrm{El}$ análisis de la $F$ de Snedecor y de la $t$ de Student indica que la correlación es real y que los coeficientes de regresión son distintos de cero con un nivel de confianza del 95\%.

El coeficiente $K_{O P}$ de la ecuación [29] representa un coeficiente de ponderación que tiene en cuenta todas las incertidumbres que pueden afectar a las variables de la ecuación [29], es decir, las incertidumbres que pueden existir en el proyecto.
Para determinar el valor de $K_{O P}$ se compararon los valores de $H T$ reales indicados en la Tabla 9, denominados $H T_{\text {real }}$, con los calculados a partir del $R O P$ obtenido por medio de la ecuación [30], designado como $H T_{\text {pred }}$. Para el resto de variables se adoptan los valores específicos de cada obra (Tabla 9).

En la Figura 3 se muestran los valores del ratio entre $H T_{\text {real }} \mathrm{y}$ $H T_{\text {pred }}$, que varía entre 0,70 y 4,68. Un valor inferior a 1 indica que emplean menos horas de las estimadas para la ejecución del tramo. El valor medio del ratio es de 1,71 con una desviación estándar de 1,138. Por tanto se recomienda un valor de 1,75 pudiendo llegar hasta 5 en el caso de obras mal definidas geológicamente.

Como se ha mencionado previamente, Hegab y Smith (15) propusieron una expresión para calcular el tiempo de retraso en la obra durante la fase de excavación del microtúnel para empresas contratistas con un rendimiento medio (ecuación [31]), tras comprobar que la distribución de Weibull representa mejor la distribución de probabilidad de retraso en la obra:

$$
D T=e^{0,011166 \cdot L+5,628}
$$

Donde $D T$ es el tiempo de retraso, expresado en minutos, y $L$ es la longitud del túnel, en metros. Como se observa, esta ecuación no tiene en cuenta ningún parámetro geomecánico del terreno a atravesar. La expresión propuesta se refiere únicamente a retrasos que se pueden producir durante la fase de excavación para los que considera 5 causas posibles: problemas con las bombas y la línea de slurry, problemas de operación, problemas con el láser, averías mecánicas y organización de los trabajos en superficie.

Utilizando el tiempo de retraso calculado mediante la ecuación [31], se puede estimar un tiempo total de obra necesario como suma del tiempo empleado en la perforación del túnel $(L / R O P)$ más los retrasos durante esa operación. Se ha calculado el valor alternativo de $K_{O P}, K_{O P}(H)$, que se obtiene como

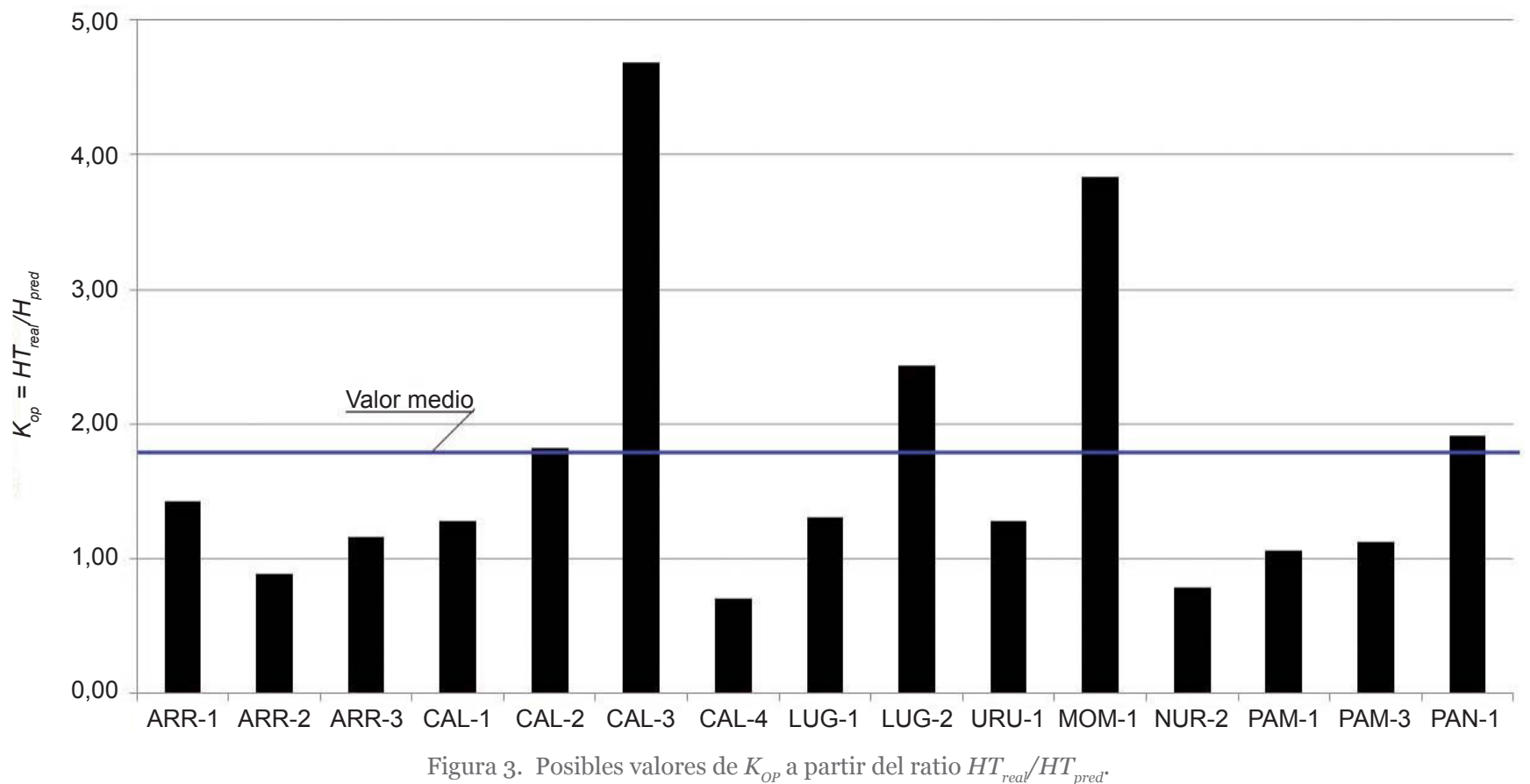


Tabla 9. Horas totales reales, horas totales obtenidas por el modelo y datos utilizados de cada uno los tramos analizados

\begin{tabular}{|c|c|c|c|c|c|c|c|c|c|c|c|c|c|c|}
\hline OBRA & $\begin{array}{c}H T_{\text {real }} \\
\text { (h) }\end{array}$ & $\begin{array}{l}H T_{\text {pred }} \\
\text { (h) }\end{array}$ & $\begin{array}{c}\mathbf{K}_{\mathrm{OP}}=\mathbf{H T}_{\text {real }} / \\
\mathbf{H T}_{\text {pred }}\end{array}$ & $\begin{array}{c}L_{T} \\
(\mathbf{m})\end{array}$ & $\begin{array}{l}T_{N T} \\
\text { (h) }\end{array}$ & $\begin{array}{l}T_{M R P} \\
\text { (h) }\end{array}$ & $\mathbf{N}_{\mathrm{cc}}$ & $\begin{array}{l}T_{M C} \\
\text { (h) }\end{array}$ & $\begin{array}{l}L_{A G} \\
(\mathbf{m})\end{array}$ & $\begin{array}{l}T_{M A G} \\
\text { (h) }\end{array}$ & $\begin{array}{l}L_{T O P} \\
\text { (m) }\end{array}$ & $\begin{array}{c}T_{M T O P} \\
\text { (h) }\end{array}$ & $\begin{array}{l}L_{M A N} \\
(\mathbf{m})\end{array}$ & $\begin{array}{c}T_{M M A N} \\
\text { (h) }\end{array}$ \\
\hline ARR-1 & 649 & 454,6 & 1,43 & 3,0 & 0,6 & 0,2 & o & 36 & 10 & 2 & 20 & 2 & 100 & 6 \\
\hline ARR-2 & 550 & 622,6 & 0,88 & 3,0 & 0,6 & 0,2 & o & 36 & 10 & 2 & 20 & 2 & 100 & 6 \\
\hline ARR-3 & 594 & 514,1 & 1,16 & 3,0 & 0,6 & 0,2 & o & 36 & 10 & 2 & 20 & 2 & 100 & 6 \\
\hline CAL-1 & 308 & 240,5 & 1,28 & 3,0 & 0,6 & 0,2 & 1 & 36 & 10 & 2 & 20 & 2 & 100 & 6 \\
\hline CAL-2 & 616 & 339,2 & 1,82 & 3,0 & 0,6 & 0,2 & 1 & 36 & 10 & 2 & 20 & 2 & 100 & 6 \\
\hline CAL-3 & 1.001 & 213,8 & 4,68 & 3,0 & 0,6 & 0,2 & o & 36 & 10 & 2 & 20 & 2 & 100 & 6 \\
\hline CAL-4 & 242 & 345,3 & 0,70 & 3,0 & 0,6 & 0,2 & o & 36 & 10 & 2 & 20 & 2 & 100 & 6 \\
\hline LUG-1 & 319 & 244,5 & 1,30 & 3,0 & 0,6 & 0,2 & o & 36 & 10 & 2 & 20 & 2 & 100 & 6 \\
\hline LUG-2 & 1.694 & 695,5 & 2,44 & 2,4 & 0,6 & 0,2 & 4 & 36 & 10 & 2 & 20 & 2 & 100 & 6 \\
\hline URU-1 & 143 & 111,7 & 1,28 & 2,4 & 0,6 & 0,2 & o & 36 & 10 & 2 & 20 & 2 & 100 & 6 \\
\hline MOM-1 & 539 & 140,6 & 3,83 & 2,4 & 0,6 & 0,2 & o & 36 & 10 & 2 & 20 & 2 & 100 & 6 \\
\hline NUR-2 & 99 & 126,1 & 0,79 & 2,4 & 0,6 & 0,2 & o & 36 & 10 & 2 & 20 & 2 & 100 & 6 \\
\hline PAM-1 & 363 & 343,4 & 1,06 & 2,4 & 0,6 & 0,2 & o & 36 & 10 & 2 & 20 & 2 & 100 & 6 \\
\hline PAM-3 & 517 & 460,3 & 1,12 & 2,4 & 0,6 & 0,2 & o & 36 & 10 & 2 & 20 & 2 & 100 & 6 \\
\hline PAN-1 & 924 & 484,7 & 1,91 & 2,4 & 0,6 & 0,2 & o & 36 & 10 & 2 & 20 & 2 & 100 & 6 \\
\hline
\end{tabular}

Nota: $N C C$ es el número de cambio de cortadores que se realizaron en el tramo. Se introduce este valor en lugar de la relación $L / L_{C}$.

cociente entre las horas totales reales, $H T_{\text {real }}$, de las obras de estudio (Tabla 9) y las horas estimadas por el método propuesto por Hegab y Smith (15) (ecuación [32]).

$$
K_{O P}(H)=\frac{H T_{\text {real }}}{\frac{L}{R O P}+D T}
$$

$R O P$ se ha obtenido mediante la ecuación [30]. En la Figura 4 se comparan los valores de $K_{O P}$ y $K_{O P}(H)$ para cada una de las obras estudiadas.

Se observa que, a excepción de 5 tramos, el valor de $K_{O P}(H)$ es siempre superior al de $K_{O P}$. Esto es debido a que el análisis que efectúan Hegab y Smith (15) sólo calcula los retrasos produci- dos durante la fase de excavación del túnel y donde las operaciones de superficie ( $5 \cdot{ }^{\mathrm{a}}$ causa) sólo suponen la causa del $5 \%$ de los tiempos de retraso. Sin embargo, tal y como se ha expuesto previamente, el ciclo de la MTBM comprende un mayor número de actividades, las cuales se llevan a cabo en superficie y que también pueden desviarse de los valores medios que se estiman. Por tanto, la formulación propuesta por Hegab y Smith (15) estima un valor menor de tiempo de obra, al tener sólo en cuenta retrasos debidos a perforación y no al resto de actividades. En consecuencia, sería necesario un $K_{O P}$ de mayor valor, un valor medio de 2,28 con respecto a 1,71, para ajustarse a las horas reales. Por tanto, se considera más conveniente aplicar la formulación propuesta en este artículo dado que tiene en cuenta todas las fases del ciclo de excavación y la variación con respecto a la realidad es menor (menor valor de $K_{O P}$ ).

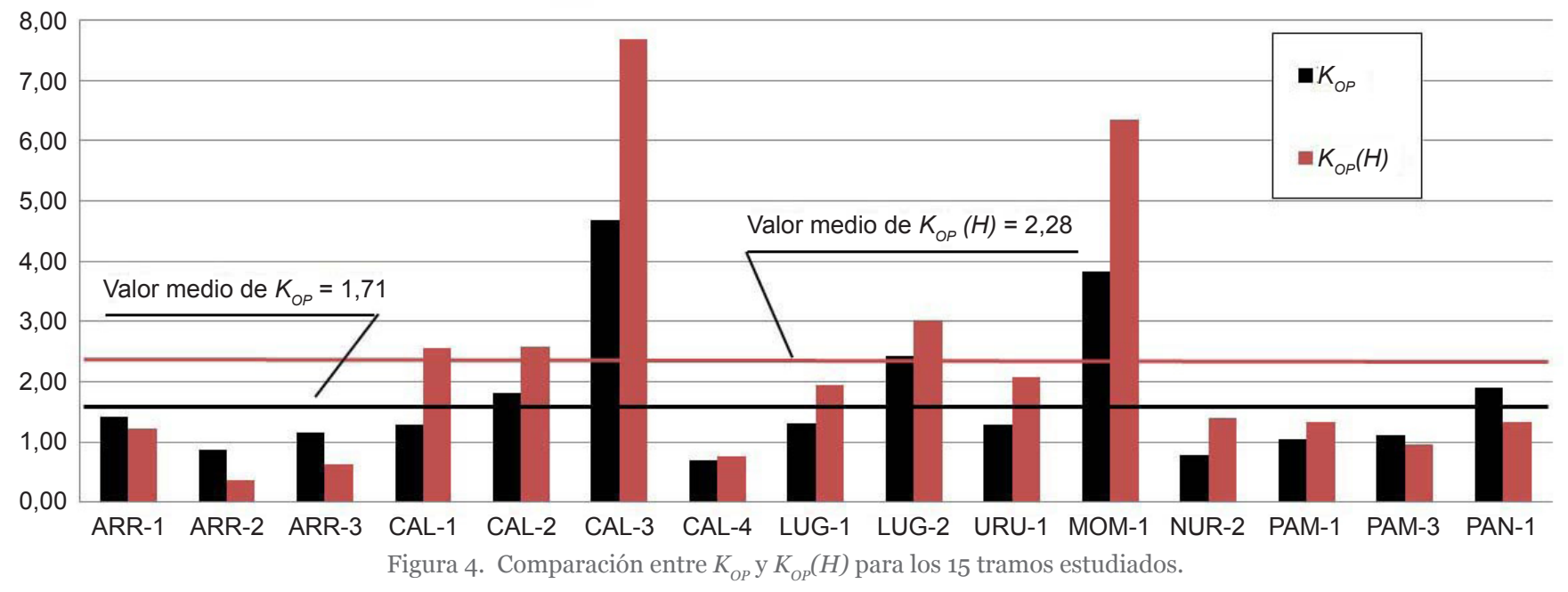


Finalmente se determina el tiempo total de obra, TTO (días), que considera desde el inicio del montaje hasta la finalización del mismo, mediante la ecuación [33]:

$$
T T O=T M+H T+T D
$$

Donde $T M$ es el tiempo de montaje necesario, con un valor habitual de 10 a 15 días, $T D$ es el tiempo empleado para el desmontaje, incluyendo la retirada de todo el cableado, bombas y conducciones del fluido de perforación al exterior, empleándose entre 10 y 15 días según la longitud del tramo, y HT es el número de horas totales empleadas en la ejecución del tramo, expresada en días según las horas trabajadas por día.

\subsection{Aplicación práctica}

En este apartado se presenta un caso práctico de aplicación de la formulación expuesta en el artículo. Se ha supuesto una actuación en la que se tiene que instalar una tubería de $250 \mathrm{~m}$, con tubos de $2,4 \mathrm{~m}$ de longitud y un diámetro nominal de $2.000 \mathrm{~mm}$. Las propiedades de la geología que debe atravesar el microtúnel se detallan en la Tabla 10, junto con los valores de las magnitudes que componen el ciclo de trabajo de la MTBM, descritos en la Tabla 8. Debido a la longitud del túnel y la geología se estima que no es necesario el cambio de los discos de corte.

Con estos datos, aplicando las ecuaciones [28] y [30], se obtiene que:

$$
\begin{gathered}
F P I=\exp (0,0193 \cdot 20+0,007 \cdot 75+1,303)= \\
=9,15 \mathrm{kN} / \mathrm{cutter}) /(\mathrm{mm} / \mathrm{rev})
\end{gathered}
$$

$$
R O P=3,289-0,019 \cdot 20-0,012 \cdot 75=3,809 \mathrm{~m} / \mathrm{h}
$$

Con el valor de $R O P$ introducido en la ecuación [29] se estima la cantidad de horas totales para realizar la obra (ecuación [36]).

$$
\begin{gathered}
H T=1,75 \cdot\left(\frac{250}{3,809}+\frac{250}{2,4} \cdot 0,6+\frac{250}{2,4} \cdot 0,2+0+\right. \\
\left.+\frac{250}{10} \cdot 2+\frac{250}{20} \cdot 2+\frac{250}{100} \cdot 6+0\right)=418,2 \mathrm{~h}
\end{gathered}
$$

Si se establece que se trabajan 8 horas diarias, el número total de días será 52,3. Si se suponen 15 días de instalación y 10 días de retirada de los equipos, mediante la ecuación [33], se logra una estimación de 77,3 días laborables para la realización de todas las actividades.

\section{CONCLUSIONES}

Los microtúneles se caracterizan por ser obras cortas, con una longitud entre los 100 y los $500 \mathrm{~m}$, en los que el presupuesto de ejecución es muy inferior a las realizadas con TBMs. Además, se trata de unas obras más dinámicas, en las que el tramo se finaliza en unos pocos meses. Por tanto, es interesante estimar cuántas horas son necesarias para la finalización de la obra y las incertidumbres que pueden modificar esta estimación.

Por otra parte, los microtúneles se realizan a profundidades bajas, donde los macizos rocosos suelen estar alterados y ser más heterogéneos, por lo que el estudio geológico-geotécnico del proyecto no puede caracterizar correctamente ni completamente el terreno debido al escaso número de sondeos que se ejecutan para la caracterización.

Tras el análisis de los datos de 15 tramos de obras de microtúnel en geologías diversas se propuso un modelo para la estimación de las horas totales de obra que serán necesarias para llevarlo a cabo, a través del cual es posible determinar los costes de alquileres, personal, energía, etc.

El modelo precisa del valor de los rendimientos de excavación. Tras observarse que los modelos propuestos para tuneladoras (TBM) no predicen con relativa aproximación los valores obtenidos en la realidad, se propone un modelo empírico para el cálculo de FPI, obtenido a partir de los datos medios de rendimiento y de parámetros geomecánicos de los 15 tramos analizados, junto con los datos en las inmediaciones de los sondeos existentes en esos 15 tramos, denominados Puntos de Control. La ecuación propuesta tiene un alto grado de correlación. Además se proporciona una estimación de la velocidad de avance lineal de la máquina, $R O P$, a partir de los mismos parámetros geomecánicos.

Tabla 10. Valores de las distintas magnitudes del caso práctico.

\begin{tabular}{|l|c|}
\hline \multicolumn{1}{|c|}{ Magnitud } & Valor \\
\hline Resistencia a compresión simple, $\sigma_{C}$ & $20 \mathrm{MPa}$ \\
\hline Rock Quality Designation, $R Q D$ & $75 \%$ \\
\hline Longitud túnel, $L$ & $250 \mathrm{~m}$ \\
\hline Longitud tubo, $L_{T}$ & $2,4 \mathrm{~m}$ \\
\hline Tiempo de instalación de nuevo tubo, $T_{N T}$ & $0,6 \mathrm{~h}$ \\
\hline Tiempo medio de reanudación de la perforación, $T_{M R P}$ & $0,2 \mathrm{~h}$ \\
\hline Número de cambio de cortadores, $N_{C C}$ & 0 \\
\hline Longitud perforable sin cambio de agua, $L_{A G}$ & $10 \mathrm{~m}$ \\
\hline Tiempo medio de cambio de agua, $T_{M A G}$ & $2 \mathrm{~h}$ \\
\hline Longitud perforable entre dos comprobaciones topográficas, $L_{T O P}$ & $20 \mathrm{~m}$ \\
\hline Tiempo empleado para comprobación topográfica, $T_{M T O P}$ & $2 \mathrm{~h}$ \\
\hline Longitud perforable sin necesidad de mantenimiento, $L_{M A N}$ & $100 \mathrm{~m}$ \\
\hline Tiempo de mantenimiento preventivo, $T_{M A N}$ & $6 \mathrm{~h}$ \\
\hline$K_{O P}$ & 1,75 \\
\hline
\end{tabular}


Se propone un coeficiente $K_{O P}$ de ponderación que aproxima el valor estimado de horas necesarias para la ejecución del microtúnel mediante el modelo a los valores reales observados en la realidad. El coeficiente $K_{O P}$ considera las incertidumbres que afectan a este tipo de proyecto, especialmente las geológicas.

Como líneas de futuras investigaciones se plantea el estudio de la variabilidad de las horas de retraso que tienen lugar en cada una de las fases del ciclo de trabajo de las MTBM, para poder identificar mejor los valores medios de los mismos. Además, se recomienda que los proyectos de microtúneles completen sus estudios geológico-geotécnicos con otros ensayos, como los de abrasividad, que permiten analizar otras características del terreno y ver si influyen en el rendimiento, de manera similar a lo que se realiza en TBMs, así como incrementar el número de sondeos y de muestras ensayadas en cada tramo.

\section{REFERENCIAS}

(1) Rostami, J., Ozdemir, L., Nilson, B. (1996, 1 de mayo). Comparison between CSM and NTH hard rock TBM performance prediction models. En Proceedings of Annual Technical Meeting of the Institute of Shaft Drilling Technology (p. 1-11). Las Vegas.

(2) Gallo Laya, J., Pérez-Acebo, H., García-Bragado, D. (2016). Excavación, sostenimiento y técnicas de corrección de túneles, obras subterráneas y labores mineras, p 99-136, Bilbao: Universidad del País Vasco UPV/EHU.

(3) Hassanpour, J., Rostami, J., Khamehchiyan, M., Bruland, A. (2009). Developing new equations for TBM performance prediction in carbonate-argillaceous rocks: a case history of Nowsood water conveyance tunnel. Geomechanics and Geoengineering: An International Journal, 4(4): 287-297, doi: http://dx.doi.org/10.1080/17486020903174303.

(4) Barton, N. (2000). TBM Tunnelling in Jointed and Faulted Rock, Rotterdam: Balkema.

(5) Bieniawski, Z. T., Celeda, B., Galera, J. M. (2007). Predicting TBM excavability. Tunnels and Tunnelling International (September): 32-35.

(6) Barton, N. (1999). TBM performance estimation in rock using $\mathrm{Q}_{\mathrm{TBM}}$. Tunnels and Tunnelling International (September): 30-34.

(7) Yagiz, S. (2006, 6 de septiembre). A model for the prediction of tunnel boring machine performance. En Proceedings of 10th IAEG Congress, paper number 383. Nottingham, UK.

(8) Bruland, A. (1998). Prediction model for performance and costs. En Norwegian TBM Tunnelling, Publication 11 (pp. 29-34). Oslo: Norwegian Tunnelling Society, NFF.

(9) Roxborough, F. F., Phillips, H. R. (1975). Rock excavation by disc cutter. International Journal of Rock Mechanics and Mining Sciences and Geomechanics Abstracts, 12(12): 361-366, doi: http://dx.doi.org/doi:10.1016/01489062(75)90547-1.

(10) Okubo, S., Fukui, K., Chen, W. (2003). Expert system for applicability of tunnel boring machines in Japan. Rock Mechanics and Rock Engineering, 36(4): 305-322, doi: http://dx.doi.org/10.1007/s00603-002-0049-6.

(11) Leitner, W., Schneider, E. (2003). Penetration prediction models for hard rock tunnel boring machines. Felsbau, 21(6): 8-13.

(12) Farrokh, E., Rostami, J., Laughton, C. (2012). Study of various models for estimation of penetration rate of hard rock TBMs. Tunnelling and Underground Space Technology, 30: 110-123, doi: http://dx.doi.org/10.1016/j.tust.2012.02.012.

(13) Stein, D. (2005). Trenchless technology for installation of cables and pipelines, Bochum, Germany: Stein \& Partner.

(14) Gesellschaft zur Forderung der Abwassertechnik e.V. (1996). Standard and Rules ATV A125E: Pipe Driving (1996) Standard ATV-A 125 E Pipe Driving. Henaf, Germany.

(15) Hegab, M. Y., Smith, G. R. (2007). Delay time analysis in microtunneling projects. Journal of Construction Engineering and Management, 133(2): 191-195, doi: http://dx.doi.org/10.1061/(ASCE)o733-9364(2007)133:2(191).

(16) Hamilton, W. H., Dollinger, G. L. (1979, 18 de junio). Optimizing tunnel boring machine and cutter design for greater boreability. En Rapid Excavation and Tunneling Conference, 1, 280-296. Atlanta: Society of Mining Engineers of the American Institute of Mining, Metallurgical and Petroleum Engineers.

(17) Nelson, P., Ingraffea, A., O'Rourke, T. (1985). TBM performance prediction using rock fracture parameters. International Journal of Rock Mechanics and Mining Sciences and Geomechanics Abstracts, 22(3): 189-192, doi: http://dx.doi. org/10.1016/0148-9062(85)93234-6.

(18) Fatemi, S. A., Ahmadi, M., Rostami, J. (2016). Evaluation of TBM performance prediction models and sensitivity analysis of input parameters. Bulletin of Engineering Geology and the Environment, 1-13, doi: http://dx.doi.org/10.1007/ s10064-016-0967-2.

(19) Graham, P. C. (1976). Rock exploration for machine manufacturers. En: Bieniawski, Z. T. (Ed.), Exploration for Rock Engineering (pp. 173-180). Johannesburg: Balkema.

(20) Farmer, I. W., Glossop, N. H. (1980). Mechanics of disc cutter penetration. Tunnels and Tunnelling, 12(6): 22-25.

(21) Cassinelli, F., Cina, S., Innaurato, N. (1982, 7 de junio). Power consumption and metal wear in tunnel-boring machines: analysis of tunnel-boring operation in hard rock. En: Tunneling 82, 3rd International Symposium (pp. 73-81). London: IMM.

(22) Innaurato, N., Mancini, A., Rondena, E., Zaninetti, A. (1991, 16 de septiembre). Forecasting and effective TBM performances in a rapid excavation of a tunnel in Italy. En 7th International Congress ISRM (pp. 1009-1014). Aachen.

(23) Rostami, J. (1997). Development of a force estimation model for rock fragmentation with disc cutters through theoretical modeling and physical measurement of crushed zone pressure (PhD thesis). Golden: Colorado School of Mines.

(24) Yagiz, S. (2008). Utilizing rock mass properties for predicting TBM performance in hard rock condition. Tunnelling and Underground Space Technology, 23(3): 326-339, doi: http://dx.doi.org/10.1016/j.tust.2007.04.011. 
(25) Gong, Q., Zhao, J. (2009). Development of a rock mass characteristics model for TBM penetration rate prediction. International Journal of Rock Mechanics and Mining Sciences, 46(1): 8-18, doi: http://dx.doi.org/10.1016/j. ijrmms.2008.03.003.

(26) Khademi Hamidi, J., Shahriar, K., Rezai, B., Rostami, J. (2010). Performance prediction of hard rock TBM using Rock Mass Rating (RMR) system. Tunnelling and Underground Space Technology, 25(4): 333-345, doi: http://dx.doi. org/10.1016/j.tust.2010.01.008.

(27) Hassanpour, J., Rostami, J., Zhao, J. (2011). A new hard rock TBM performance prediction model for project planning. Tunnelling and Underground Space Technology, 26(5): 595-603, doi: http://dx.doi.org/10.1016/j.tust.2011.04.004. 